\title{
PAPÉIS DE LIDERANÇA EM ENFERMAGEM PREDITORES DA SEGURANÇA DO DOENTE NO BLOCO OPERATÓRIO
}

\author{
PREDICTING NURSING LEADERSHIP \\ ROLES OF PATIENT SAFETY IN THE \\ OPERATING ROOM
}

\section{ROLES DE LIDERAZGO DE ENFERMERÍA QUE PREDICEN LA SEGURIDAD DEL PACIENTE EN EL QUIRÓFANO}

\author{
Ana Sofia de Carvalho Mota \\ Amélia Filomena de Oliveira Mendes Castilho \\ Maria Manuela Ferreira Pereira Martins ${ }^{3}$
}

\begin{abstract}
Como citar este artigo: Mota ASC, Castilho AFOM, Martins MMFP. Papéis de liderança em enfermagem preditores da segurança do doente no bloco operatório. Rev baiana enferm. 2022;36:e46571.

Objetivo: identificar a liderança percecionada pelos enfermeiros perioperatórios e determinar os papéis de liderança preditores da segurança do doente no bloco operatório. Método: estudo descritivo e explicativo numa amostra de 1.001 enfermeiros, com recurso ao questionário de segurança do doente no bloco operatório e ao instrumento de liderança de Quinn adaptado à saúde. No tratamento de dados utilizou-se a aplicação IBM SPSS Statistics, versão 25.0. Resultado: todas as dimensões de liderança obtiveram valor superior ao ponto médio da escala $(M>4)$. Observado um valor mínimo no papel de Inovador $(M=4,63)$ e máximo no Produtor $(M=5,04)$. Os modelos de regressão permitiram salientar que os papéis de liderança são preditores da segurança do doente no bloco operatório, destacando-se os papéis Monitor, Produtor e Facilitador. Conclusão: os enfermeiros perioperatórios têm perceção moderadamente positiva do exercício da liderança, indiciando a necessidade de se promover o desenvolvimento dos diferentes papéis de liderança exercidos pelos enfermeiros gestores perioperatórios.
\end{abstract}

Descritores: Liderança. Segurança do Paciente. Salas Cirúrgicas. Enfermagem Perioperatória. Cuidados Perioperatórios.

Objective: to identify the leadership perceived by perioperative nurses and to determine the role of leadership predictors of patient safety in the operating room. Method: descriptive and explanatory study in a sample of 1,001 nurses, using the patient safety questionnaire in the operating room and Quinn's leadership instrument adapted to bealth. The IBM SPSS Statistics application, version 25.0, was used in the data processing. Result: all leadership dimensions were higher than the midpoint of the scale $(M>4)$. A minimum value was observed in the role of Innovator $(M=4.63)$ and maximum in the Producer $(M=5.04)$. The regression models allowed pointing out that leadership roles are predictors of patient safety in the operating room, especially the roles Monitor, Producer and Facilitator. Conclusion:

Enfermeira. Mestre em Enfermagem Médico-cirúrgica. Enfermeira Gestora do Bloco Operatório do Centro Cirúrgico de Coimbra. Coimbra, Portugal. anasofiacarvalhomota@esenfc.pt. https://orcid.org/0000-0002-3547-076I.

2 Enfermeira. Doutora em Ciências de Enfermagem. Professora Adjunta da Escola Superior de Enfermagem de Coimbra. Coimbra, Portugal. https://orcid.org/00000002-4420-8861.

Enfermeira. Doutora em Ciências de Enfermagem. Professora Coordenadora da Escola Superior de Enfermagem do Porto. Porto, Portugal. https://orcid.org/00000003-1527-9940 
perioperative nurses have moderately positive perception of leadership exercise, indicating the need to promote the development of different leadership roles exercised by perioperative management nurses.

Descriptors: Leadership. Patient Safety. Operating Rooms. Perioperative Nursing. Perioperative Care.

Objetivo: identificar el liderazgo percebido por las enfermeras perioperatorias y determinar el papel de los predictores de liderazgo de la seguridad del paciente en el quirófano. Método: estudio descriptivo y explicativo en una muestra de 1.001 enfermeras, utilizando el cuestionario de seguridad del paciente en el quirófano y el instrumento de liderazgo de Quinn adaptado a la salud. La aplicación IBM SPSS Statistics, versión 25.0, se utilizó en el procesamiento de datos. Resultado: todas las dimensiones de liderazgo fueron superiores al punto medio de la escala $(M>4)$. Se observó un valor mínimo en el rol de Innovador $(M=4,63)$ y máximo en el Productor $(M=5,04)$. Los modelos de regresión permitieron señalar que los roles de liderazgo son predictores de la seguridad del paciente en el quirófano, especialmente los roles Monitor, Productor y Facilitador. Conclusión: las enfermeras perioperatorias tienen una percepción moderadamente positiva del ejercicio de liderazgo, indicando la necesidad de promover el desarrollo de diferentes roles de liderazgo ejercidos por las enfermeras administradoras de quirofano.

Descriptores: Liderazgo. Seguridad del paciente. Quirófanos. Enfermería Perioperatoria. Cuidados perioperatorios.

\section{Introdução}

A segurança do doente (SD) é assumida como prioridade pelas organizações de saúde e pelas instâncias nacionais e internacionais que regulam este setor ${ }^{(1-2)}$. Não obstante os inúmeros esforços de sensibilização e melhoria realizados nas duas últimas décadas, continuam a registrar-se números muito elevados de incidentes no âmbito da prestação de cuidados, reconhecendo-se que o progresso na área da SD tem sido demasiado lento ${ }^{(3)}$. O contexto complexo no qual se desenvolve a prestação de cuidados exige uma liderança efetiva a fim de promover um compromisso dos profissionais com a qualidade e a segurança dos cuidados ${ }^{(2)}$. Os enfermeiros constituem a maior força de trabalho das organizações hospitalares $^{(4)}$ e têm forte influência sobre a $\mathrm{SD}^{(5-6)}$, consequentemente, a ação dos líderes também se reflete nesse domínio ${ }^{(7)}$. A evidência indica que uma liderança de enfermagem efetiva tem sido associada a menor número de eventos adversos, erros de medicação, menor mortalidade, taxas mais baixas de infeções associadas aos cuidados de saúde, menor taxa de úlceras de pressão e de quedas e melhorias no ambiente da prática de cuidados ${ }^{(7-10)}$.

A Organização Mundial da Saúde $(\mathrm{OMS})^{(11)}$ defende que, para a implementação efetiva e sustentada de projetos de melhoria da SD, é fundamental que, localmente, esses sejam liderados com eficiência. Partindo dessa premissa, reconhecendo o bloco operatório (BO) como local comum de ocorrência de eventos adversos ${ }^{(12-13)} \mathrm{e}$ a existência de uma lacuna de estudos no âmbito da liderança em contexto de BO, é fundamental identificar a liderança percecionada pelos enfermeiros perioperatórios e determinar os papéis de liderança preditores da SD no BO.

$\mathrm{O}$ interesse crescente pela liderança tem direcionado a investigação para características da prática de liderança que garantam sucesso nos resultados organizacionais. Quinn e Roughbaug conceptualizaram um modelo de liderança, o Modelo dos Valores Contrastantes ${ }^{(14)}$. Esse modelo é um modelo diferenciado e privilegiado, por se basear em várias teorias de liderança que contemplam diferentes papéis ${ }^{(15)}$. O Modelo dos Valores Contrastantes tem por base quatro modelos (Modelo das Relações Humanas, dos Sistemas Abertos, dos Objetivos Racionais e dos Processos Internos) repartidos em oito papéis, que se inscrevem nas duas dimensões-chave da liderança: flexibilidade/estabilidade e orientação externa/interna ${ }^{(16)}$. Essas dimensões-chave formam quatro quadrantes, onde se distribuem os diferentes modelos ${ }^{(14)}$. O Modelo das Relações Humanas localiza-se no quadrante da flexibilidade e orientação interna, sendo representado pelos papéis de Mentor e Facilitador ${ }^{(14)}$. Esse 
modelo parte do princípio que o envolvimento resulta em compromisso, dando ênfase à participação, à resolução de conflitos e à criação de consensos, sendo orientado para as equipas ${ }^{(16)}$. O líder deve, assim, tornar-se num facilitador centrado nos processos e num mentor empático ${ }^{(15)}$. Os Facilitadores são orientados para o processo, promovendo esforços coletivos, coesão, trabalho em equipa e gestão de conflitos. Os Mentores focam-se no desenvolvimento das pessoas, procurando exercer uma orientação empática, uma escuta ativa, apoiando individualmente os liderados no seu desenvolvimento de competências ${ }^{(17)}$.

O Modelo dos Sistemas Abertos localiza-se no quadrante da flexibilidade e orientação externa, integrando os papéis de Inovador e Broker ${ }^{(14)}$. Esse modelo parte da premissa que a adaptação e a inovação contínuas levam à aquisição e manutenção de recursos externos ${ }^{(15)}$. Emerge da necessidade de liderança num mundo em constante mudança e com grande desenvolvimento de conhecimento ${ }^{(15)}$. Esse contexto exige que o líder seja um inovador criativo e um negociador que exerce a sua influência na organização ${ }^{(14)}$. Os Inovadores caracterizam-se por serem visionários, gestores da mudança, integradores da inovação e criativos na resolução de problemas. Os Brokers representam os líderes influentes, que exercem poder, que criam e mantêm uma base de poder, uma ligação com o exterior e apresentam uma habilidade negociadora que garante a obtenção dos recursos ${ }^{(17)}$.

O Modelo de Objetivos Racionais localiza-se no quadrante do controlo e orientação externa, integrando os papéis de Diretor e Produtor ${ }^{(14)}$. Esse modelo é mais focado na produtividade e no lucro, assenta no princípio que uma direção clara leva a resultados positivos ${ }^{(15)}$. O líder deve ser, assim, um produtor pragmático e um diretor que decide ${ }^{(15)}$. Os Diretores definem metas, planificam e estabelecem objetivos, tomam iniciativas e delegam de forma eficaz. Os Produtores são centrados nas tarefas, são motivados e motivam os liderados a aceitarem responsabilidades e manterem uma produtividade elevada ${ }^{(17)}$.

Por fim, o Modelo dos Processos Internos localiza-se no quadrante do controlo e orientação interna, integrando os papéis de Monitor e Coordenador $^{(14)}$. Esse modelo dá ênfase à definição de responsabilidades, mensuração e documentação, assentando na premissa que a normalização leva à estabilidade ${ }^{(15)}$. Como Monitores, os líderes devem estar informados do que se passa na unidade, assegurar-se que os liderados cumprem as normas e atinjem os objetivos. Como Coordenadores, dão ênfase à organização e à coordenação dos esforços da equipa, minimizando disfunções e conflitos ${ }^{(17)}$. O líder eficaz é o que desempenha simultaneamente os oito papéis de liderança, contemplando a complexidade que caracterizam as organizações de saúde ${ }^{(15)}$.

Dessa forma, este estudo tem como objetivo identificar a liderança percecionada pelos enfermeiros perioperatórios e determinar os papéis de liderança preditores da segurança do doente no bloco operatório.

\section{Método}

O presente estudo, descritivo e explicativo, está inserido numa investigação inicial ${ }^{(18-19)}$, utilizando a mesma amostra e os procedimentos ético-legais. A população-alvo é constituída por 2.975 enfermeiros perioperatórios que exercem funções em BOs (de doentes adultos) de hospitais do Serviço Nacional de Saúde com valências cirúrgicas, incluídos nos grupos de benchemarking da Administração Central do Sistema de Saúde ${ }^{(20)}$. Para maior representatividade da amostra foram selecionados hospitais de cada grupo de benchemarking e Administrações Regionais de Saúde (ARS) tendo sido realizada uma amostragem por clusters. Integraram a amostra 24 hospitais, com uma percentagem por grupo de benchemarking entre 50\% (grupos B e E) e $66,7 \%$ (grupo F) e por ARS entre 42,86\% (Lisboa e Vale do Tejo) e 100\% (Algarve), correspondendo ao total de 46 BOs. Como critério de inclusão na amostra definiu-se ter tempo de atividade profissional superior a 6 meses. Como critérios de exclusão adotou-se: exercer funções como enfermeiro(a) gestor(a) e estar ausente temporariamente do serviço no período da colheita de dados por atestado médico, licença de férias ou outra licença. O período de colheita de dados 
ocorreu entre janeiro e outubro de 2018. Foram entregues 1.798 questionários, abrangendo todos os enfermeiros que cumpriam os critérios de inclusão, obtendo-se taxa de resposta de 55,70\%. A amostra, constituída por 1.001 participantes, possui um erro amostral estimado de 2,6\%. Os enfermeiros inquiridos são, maioritariamente, do sexo feminino $(84,90 \%)$, com média de idade de 42,74 anos (DP = 0,27) e uma média 19,76 anos ( $\mathrm{DP}=0,27)$ de tempo de exercício. Os participantes possuem tempo de experiência em BO e no atual serviço, respetivamente, de 13,52 anos $(\mathrm{DP}=0,28)$ e 11,56 anos $(\mathrm{DP}=0,27)$. No que se refere ao grau académico, a maioria é licenciada (79,10\%), 18,50\% são mestres, 1,90\% são bacharéis e 0,50\% são doutorados. Apenas 17,90\% possuem o título de especialista. A maioria dos enfermeiros perioperatórios trabalha em BOs centrais (76,9\%), 15,60\% trabalham em BOs de cirurgia de ambulatório e 7,40\% em BOs periféricos. Mais da metade trabalha em BOs acreditados/certificados (59,7\%).

Como instrumento de colheita de dados utilizou-se o questionário de Segurança do Doente no $\mathrm{BO}(\mathrm{SDBO})^{(18-19)}$ e o instrumento de Liderança de Quinn ${ }^{(14,21)}$ adaptado à área da saúde ${ }^{(17)}$. O questionário SDBO é constituído por 79 itens, pontuáveis numa escala tipo Likert de 1 (nunca) a 5 (sempre), que permite avaliar 19 dimensões de SD, nomeadamente: Cultura de Segurança do ambiente interno (D1), Segurança da comunicação - boas práticas (D2), Segurança da comunicação - auditorias (D3), Segurança cirúrgica - boas práticas (D4), Segurança cirúrgica - auditorias (D5), Segurança na utilização da medicação - boas práticas (D6), Segurança na utilização da medicação - auditoria (D7), Segurança na utilização da medicação - prescrição (D8), Identificação inequívoca dos doentes - boas práticas (D9), Identificação inequívoca dos doentes - auditorias (D10), Prevenção de quedas - boas práticas (D11), Prevenção de quedas auditorias (D12), Prevenção de Úlceras de Pressão - boas práticas (D13), Prevenção de Úlceras de Pressão - auditorias (D14), Prevenção de Úlceras de Pressão - recursos (D15), Notificação de incidentes (D16), Análise e prevenção de incidentes (D17), Prevenção e controlo de infeção e resistência aos antimicrobianos (PCIRA) - boas práticas (D18) e PCIRA - formação e vigilância epidemiológica (D19). Esse questionário apresentou valores psicométricos adequados, com valores de alfa de Cronbach entre 0,66 e 0,98 nas 19 dimensões ${ }^{(18)}$. A maioria dos itens, com duas exceções, apresentaram níveis de saturação no fator $\geq 0,48$ e valores de correlação corrigida de cada item com a dimensão entre 0,41 e 0,96. A validade convergente-discriminante dos itens revelou maior correlação dos itens com o total da dimensão a que pertencem do que com a dimensão a que não pertencem. O instrumento de Liderança de Quinn adaptada à área da saúde ${ }^{(17)}$ é composto por 32 itens que avaliam as competências de liderança, com base nas perceções dos liderados, distribuídas por 8 papéis: Mentor, Facilitador, Broker, Inovador, Monitor, Coordenador, Diretor e Produtor. Os itens encontram-se agrupados numa escala tipo Likert com sete opções de resposta que variam entre "quase nunca" (1) e "quase sempre" (7). O ponto médio da escala corresponde ao valor quatro, sendo que valores maiores ou iguais refletem uma perceção da liderança positiva. Esse instrumento foi utilizado em vários estudos nacionais ${ }^{(15,17,22-23)}$, tendo evidenciado boas características psicométricas.

Foi solicitado parecer à Comissão de Ética da Unidade de Investigação em Ciências da Saúde da Enfermagem da Escola Superior de Enfermagem de Coimbra e realizados pedidos de autorização de recolha de informação aos conselhos de administração (CA) dos hospitais envolvidos no estudo. Obteve-se parecer positivo (P 458-09-2017) por parte da Comissão de Ética supracitada e dos CA dos 24 hospitais. Posteriormente foram realizadas reuniões com os enfermeiros gestores dos diferentes BOs, para explicar o objetivo do estudo e solicitar a sua colaboração na distribuição dos questionários, entregues em envelopes abertos com explicitação dos objetivos e solicitação de consentimento informado. A devolução do questionário foi realizada em envelope fechado e o consentimento informado recolhido em separado. 
No tratamento de dados utilizou-se o programa informático IBM SPSS Statistics, versão 25.0. Na análise dos dados recorreu-se à estatística descritiva e procedeu-se à análise do modelo de regressão linear múltipla, com base no método stepwise. Optou-se por esse método por permitir identificar as variáveis com maior poder preditor, eliminando as supérfluas. Na análise descritiva calcularam-se as frequências (absolutas e percentuais), as medidas de tendência central (média, máximo e mínimo) e as medidas de dispersão (desvio-padrão (DP)). Para avaliação da fiabilidade calculou-se a consistência interna de cada dimensão pelo coeficiente Alfa de Cronbach.

\section{Resultados}

Inicialmente foi realizado análise descritiva dos itens que compõem a escala de liderança de Quinn $^{(17)}$. Como se pode observar na Tabela 1, todos os itens obtiveram valores entre o mínimo e o máximo, evidenciando heterogeneidade das respostas e uma dispersão apreciável (DP mínimo $\geq 1,30$ ), indicadora do poder discriminativo. Os valores médios mais elevados significam melhor perceção do exercício da liderança pelo superior hierárquico. Numa apreciação global constatou-se que todos os itens possuíam valores médios superiores ao ponto médio da escala (4). Os itens que obtiveram valores mais positivos foram: Tem acesso a pessoas de nível mais elevado (item 18; M=5,62; DP=1,30), Mantém-se ao corrente do que se passa na unidade (item 21; $\mathrm{M}=5,40 ; \mathrm{DP}=1,46$ ), Trabalha com informação técnica (item 17; M=5,24; DP=1,45) e Define áreas de responsabilidade para os subordinados (item 7; $M=5,24 ; D P=1,55$ ). Por outro lado, os itens Propõe ideias criativas (item 1; $\mathrm{M}=4,20$; $\mathrm{DP}=1,60)$; Compara registos e relatórios na procura de discrepâncias (item 14; M=4,44; $\mathrm{DP}=1,68)$ e Apresenta ideias novas aos superiores de modo persuasivo (item 27; $M=4,48$; $\mathrm{DP}=1,61$, obtiveram valores médios mais baixos.

Tabela 1 - Análise descritiva dos itens da escala de Liderança: mínimo, máximo, média e desvio-padrão. Coimbra, Portugal - 2018. (N = 1.001)

(continua)

\begin{tabular}{|c|c|c|c|c|c|}
\hline Itens & $\mathbf{n}$ & Mínimo & Máximo & Média & $\begin{array}{l}\text { Desvio- } \\
\text {-Padrão }\end{array}$ \\
\hline 1. Propõe ideias criativas & 998 & 1 & 7 & 4,20 & 1,60 \\
\hline $\begin{array}{l}\text { 2. Promove a continuidade das operações diárias da } \\
\text { unidade }\end{array}$ & 994 & 1 & 7 & 5,04 & 1,54 \\
\hline 3. Exerce influência positiva na unidade & 998 & 1 & 7 & 4,65 & 1,62 \\
\hline $\begin{array}{l}\text { 4. Revê cuidadosamente e de forma detalhada toda a } \\
\text { informação disponível }\end{array}$ & 998 & 1 & 7 & 4,89 & 1,52 \\
\hline $\begin{array}{l}\text { 5. Mantém na unidade uma orientação para os } \\
\text { resultados }\end{array}$ & 994 & 1 & 7 & 4,89 & 1,58 \\
\hline $\begin{array}{l}\text { 6. Facilita a construção de consensos no trabalho da } \\
\text { unidade }\end{array}$ & 998 & 1 & 7 & 4,67 & 1,58 \\
\hline $\begin{array}{l}\text { 7. Define áreas de responsabilidade para os } \\
\text { subordinados }\end{array}$ & 999 & 1 & 7 & 5,24 & 1,55 \\
\hline 8. Ouve os problemas pessoais dos subordinados & 998 & 1 & 7 & 4,96 & 1,66 \\
\hline 9. Minimiza as perturbações no fluxo de trabalho & 998 & 1 & 7 & 4,69 & 1,59 \\
\hline $\begin{array}{l}\text { 10. Utiliza/experimenta conceitos e procedimentos } \\
\text { novos }\end{array}$ & 997 & 1 & 7 & 4,69 & 1,58 \\
\hline 11. Encoraja a tomada de decisão participativa & 997 & 1 & 7 & 4,69 & 1,64 \\
\hline $\begin{array}{l}\text { 12. Assegura que todos saibam os objetivos da } \\
\text { unidade }\end{array}$ & 997 & 1 & 7 & 5,01 & 1,64 \\
\hline 13. Influencia decisões tomadas em níveis superiores & 991 & 1 & 7 & 4,60 & 1,59 \\
\hline $\begin{array}{l}\text { 14. Compara registos e relatórios na procura de } \\
\text { discrepâncias }\end{array}$ & 983 & 1 & 7 & 4,44 & 1,68 \\
\hline 15. Verifica se a unidade cumpre os objetivos & 990 & 1 & 7 & 5,10 & 1,56 \\
\hline
\end{tabular}


Tabela 1 - Análise descritiva dos itens da escala de Liderança: mínimo, máximo, média e desvio-padrão. Coimbra, Portugal - 2018. $(\mathrm{N}=1.001)$ (conclusão)

\begin{tabular}{|c|c|c|c|c|c|}
\hline Itens & $\mathbf{n}$ & Mínimo & Máximo & Média & $\begin{array}{l}\text { Desvio- } \\
\text {-Padrão }\end{array}$ \\
\hline $\begin{array}{l}\text { 16. Demonstra empatia e preocupação no } \\
\text { relacionamento com os subordinados }\end{array}$ & 999 & 1 & 7 & 4,84 & 1,69 \\
\hline 17. Trabalha com informação técnica & 996 & 1 & 7 & 5,24 & 1,45 \\
\hline 18. Tem acesso a pessoas de nível mais elevado & 991 & 1 & 7 & 5,62 & 1,30 \\
\hline $\begin{array}{l}\text { 19. Estabelece objetivos claros para o trabalho na } \\
\text { unidade }\end{array}$ & 999 & 1 & 7 & 4,94 & 1,56 \\
\hline 20. Trata as pessoas de modo delicado e cuidado & 998 & 1 & 7 & 4,99 & 1,66 \\
\hline $\begin{array}{l}\text { 21. Mantém-se ao corrente do que se passa na } \\
\text { unidade }\end{array}$ & 998 & 1 & 7 & 5,40 & 1,46 \\
\hline 22. Resolve problemas com criatividade e inteligência & 999 & 1 & 7 & 4,81 & 1,59 \\
\hline $\begin{array}{l}\text { 23. Impulsiona a unidade para o cumprimento dos } \\
\text { seus objetivos }\end{array}$ & 997 & 1 & 7 & 5,17 & 1,53 \\
\hline $\begin{array}{l}\text { 24. Encoraja os subordinados a partilhar ideias em } \\
\text { grupo }\end{array}$ & 998 & 1 & 7 & 4,72 & 1,67 \\
\hline 25. Procura inovações e melhorias potenciais & 998 & 1 & 7 & 4,82 & 1,60 \\
\hline 26. Clarifica prioridades e direções & 996 & 1 & 7 & 4,85 & 1,56 \\
\hline $\begin{array}{l}\text { 27. Apresenta ideias novas aos superiores de modo } \\
\text { persuasivo }\end{array}$ & 992 & 1 & 7 & 4,48 & 1,61 \\
\hline 28. Proporciona à unidade uma sensação de ordem & 998 & 1 & 7 & 4,63 & 1,72 \\
\hline $\begin{array}{l}\text { 29. Demonstra preocupação com as necessidades } \\
\text { dos subalternos }\end{array}$ & 999 & 1 & 7 & 4,65 & 1,75 \\
\hline $\begin{array}{l}\text { 30. Enfatiza a realização dos objetivos propostos pela } \\
\text { unidade }\end{array}$ & 996 & 1 & 7 & 4,96 & 1,60 \\
\hline 31. Desenvolve trabalho em equipa & 999 & 1 & 7 & 4,77 & 1,67 \\
\hline 32. Analisa planos escritos e projetos & 993 & 1 & 7 & 4,63 & 1,69 \\
\hline
\end{tabular}

Fonte: Elaboração própria.

As dimensões da escala de liderança foram calculadas conforme proposta do autor da versão adaptada à area da saúde ${ }^{(17)}$ e apresentaram valores de alfa de Cronbach que variaram entre 0,87 (Broker) e 0,94 (Facilitador), indiciando boa consistência interna. A análise das medidas de tendência central e o valor do desvio-padrão, observados nas oito dimensões permite salientar o bom poder discriminativo de cada dimensão (DP $\geq 1,30$ ) (Tabela 2). Constatou-se heterogeneidade nas respostas obtidas em todos os pontos da escala em todos os itens. A perceção de liderança é globalmente positiva, observando-se, em todas as dimensões, valores médios superiores ao ponto médio da escala ( $>4$ ). Contudo, constatou-se que os profissionais têm uma perceção média mais elevada nos papéis de Produtor $(\mathrm{M}=5.07 ; \mathrm{DP}=1,41)$ e Diretor $(\mathrm{M}=5.01 ; \mathrm{DP}=1,39)$. Os papéis de Inovador e Facilitador foram percecionados com menor ênfase $(M=4.63 ; \mathrm{DP}=1,43$ e $\mathrm{M}=4.72 ; \mathrm{DP}=1,50)$, respetivamente.

Tabela 2 - Análise descritiva e alfa de Cronbach das dimensões da escala de Liderança: itens, mínimo, máximo, média e desvio-padrão. Coimbra, Portugal - 2018. ( $\mathrm{N}=1.001)$

\begin{tabular}{l|c|c|c|c|c|c|c}
\hline Dimensões & Itens & $\mathbf{n}$ & $\begin{array}{c}\text { Alfa de } \\
\text { Cronbach }\end{array}$ & Mínimo & Máximo & Média & $\begin{array}{c}\text { Desvio- } \\
\text {-Padrão }\end{array}$ \\
\hline Papel de Broker & $3 ; 13 ; 18 ; 27$ & 981 & 0,87 & 1 & 7 & 4,85 & 1,30 \\
Papel de Inovador & $1 ; 10 ; 22 ; 25$ & 995 & 0,92 & 1 & 7 & 4,63 & 1,43 \\
Papel de Produtor & $5 ; 15 ; 23 ; 30$ & 982 & 0,92 & 1 & 7 & 5,04 & 1,41 \\
Papel de Diretor & $7 ; 12 ; 19 ; 26$ & 994 & 0,91 & 1 & 7 & 5,01 & 1,39
\end{tabular}


Tabela 2 - Análise descritiva e alfa de Cronbach das dimensões da escala de Liderança: itens, mínimo, máximo, média e desvio-padrão. Coimbra, Portugal - 2018. ( N = 1.001)

\begin{tabular}{l|c|c|c|c|c|c|c}
\hline Dimensões & Itens & $\mathbf{n}$ & $\begin{array}{c}\text { Alfa de } \\
\text { Cronbach }\end{array}$ & Mínimo & Máximo & Média & $\begin{array}{c}\text { Desvio- } \\
\text {-Padrão }\end{array}$ \\
\hline $\begin{array}{l}\text { Papel de } \\
\text { Coordenador }\end{array}$ & $2 ; 9 ; 21 ; 28$ & 991 & 0,89 & 1 & 7 & 4,94 & 1,37 \\
Papel de Monitor & $4 ; 14 ; 17 ; 32$ & 977 & 0,89 & 1 & 7 & 4,80 & 1,38 \\
Papel de Mentor & $8 ; 16 ; 20 ; 29$ & 997 & 0,93 & 1 & 7 & 4,86 & 1,54 \\
Papel de Facilitador & $6 ; 11 ; 24 ; 31$ & 995 & 0,94 & 1 & 7 & 4,72 & 1,50 \\
\hline
\end{tabular}

Fonte: Elaboração própria.

Com o intuito de analisar em que medida a SD no $\mathrm{BO}$ pode ser explicada pelos papéis de liderança, recorreu-se à técnica de regressão linear múltipla. Na análise considerou-se como variáveis dependentes as 19 dimensões do questionário de SDBO e como variáveis preditoras as 8 dimensões de liderança ${ }^{(17)}$. Na Tabela 3 é apresentada uma síntese dos resultados dos papéis de liderança que entram nos modelos preditores das dimensões de SD no BO. Todos os papéis de liderança são preditores da SD no BO, com exceção do papel de Broker. Os modelos apresentaram maior poder explicativo nas dimensões: Prevenção e controlo de infeção e resistências aos antimicrobianos - formação e vigilância epidemiológica (35\%), Segurança cirúrgica - auditorias (32\%), Cultura de segurança do ambiente interno (30\%), Segurança da comunicação - auditorias (27\%) e Segurança cirúrgica - boas práticas (27\%). Por outro lado, revelaram menor poder explicativo nas dimensões Segurança na utilização da medicação - prescrição (8\%), Prevenção de úlceras de pressão - boas práticas (9\%), Prevenção de úlceras de pressão recursos (10\%) e Notificação de incidentes (12\%). Destacou-se o efeito preditor positivo dos papéis de Monitor, Produtor e Facilitador, uma vez que entraram na maioria dos modelos explicativos, com valores de Beta positivos, que indiciam elevado peso explicativo. Salienta-se a relação inversa do papel de Mentor, com valores negativos de Beta (Tabela 3).

Tabela 3 - Dimensões da liderança que entram nos modelos preditores das dimensões de Segurança do Doente no Bloco Operatório: pesos da regressão Beta ajustado, testes t e percentagem explicativa de cada modelo. Coimbra, Portugal - 2018. $(\mathrm{N}=1.001)$

\begin{tabular}{|c|c|c|c|c|c|}
\hline $\begin{array}{l}\text { Dimensões } \\
\text { Segurança do Doente no Bloco Operatório }\end{array}$ & $\begin{array}{c}\text { Liderança } \\
\text { (Dimensões } \\
\text { Preditoras) }\end{array}$ & $\begin{array}{c}\text { Pesos da } \\
\text { regressão } \\
\text { Beta } \\
\text { ajustado }\end{array}$ & $\begin{array}{c}\text { Teste } \\
\mathbf{T}\end{array}$ & $\mathbf{p}^{*}$ & $\begin{array}{c}\% \\
\text { explicada }\end{array}$ \\
\hline \multirow[t]{4}{*}{ D1 Cultura de segurança interna } & Produtor & 0,29 & 4,28 & - & \multirow[t]{4}{*}{$30 \%$} \\
\hline & Monitor & 0,23 & 3,29 & - & \\
\hline & Mentor & $-0,25$ & $-4,17$ & - & \\
\hline & Facilitador & 0,25 & 3,27 & - & \\
\hline \multirow{3}{*}{$\begin{array}{l}\text { D2 Segurança da comunicação - boas } \\
\text { práticas }\end{array}$} & Produtor & 0,34 & 4,83 & - & \multirow[t]{3}{*}{$17 \%$} \\
\hline & Mentor & $-0,18$ & $-3,90$ & - & \\
\hline & Monitor & 0,21 & 2,90 & - & \\
\hline \multirow[t]{5}{*}{ D3 Segurança da comunicação - auditorias } & Inovador & 0,28 & 3,45 & - & \multirow[t]{5}{*}{$27 \%$} \\
\hline & Produtor & 0,11 & 1,48 & 0,04 & \\
\hline & Mentor & $-0,26$ & $-4,41$ & - & \\
\hline & Facilitador & 0,23 & 2,55 & 0,01 & \\
\hline & Monitor & 0,15 & 1,98 & 0,05 & \\
\hline \multirow[t]{2}{*}{ D4 Segurança cirúrgica - boas práticas } & Monitor & 0,31 & 4,50 & - & \multirow[t]{2}{*}{$27 \%$} \\
\hline & Produtor & 0,17 & 2,52 & 0,01 & \\
\hline
\end{tabular}


Tabela 3 - Dimensões da liderança que entram nos modelos preditores das dimensões de Segurança do Doente no Bloco Operatório: pesos da regressão Beta ajustado, testes t e percentagem explicativa de cada modelo. Coimbra, Portugal - 2018. $(\mathrm{N}=1.001)$

(conclusão)

\section{Dimensões Segurança do Doente no Bloco Operatório}

D5 Segurança cirúrgica - auditorias

D6 Segurança na utilização de medicação boas práticas

D7 Segurança na utilização de medicação auditorias

D8 Segurança na utilização da medicação prescrição

D9 Identificação inequívoca - boas práticas

D10 Identificação inequívoca - auditorias

D11 Prevenção de quedas - boas práticas

D12 Prevenção de quedas - auditorias

D13 Prevenção de Úlceras de Pressão - boas práticas

D14 Prevenção de Úlceras de Pressão auditorias

D15 Prevenção de Úlceras de Pressão recursos

D16 Notificação de incidentes

D17 Análise e prevenção de incidentes D18 Prevenção e Controlo de Infeção e Resistências aos Antimicrobianos - boas práticas

D19 Prevenção e Controlo de Infeção e Resistências aos Antimicrobianos - formação e Vigilância Epidemiológica

\begin{tabular}{|c|} 
Liderança \\
(Dimensões \\
Preditoras)
\end{tabular}

Monitor

Produtor

Mentor

Facilitador

Produtor

Coordenador

Inovador

Inovador

Produtor

Diretor

Monitor

Produtor

Facilitador

Coordenador

Monitor

Inovador

Mentor

Facilitador

Diretor

Monitor

Monitor

Mentor

Facilitador

Inovador

Monitor

Monitor

Mentor

Facilitador

Monitor

Monitor

Produtor

Produtor

Monitor

Facilitador

Diretor

Inovador

Monitor

Produtor

Mentor

Facilitador

\begin{tabular}{|c|c|c|c|}
\hline $\begin{array}{l}\text { Pesos da } \\
\text { regressão } \\
\text { Beta } \\
\text { ajustado }\end{array}$ & $\begin{array}{c}\text { Teste } \\
\mathbf{T}\end{array}$ & $\mathbf{p}^{*}$ & $\begin{array}{c}\% \\
\text { explicada }\end{array}$ \\
\hline 0,29 & 4,09 & - & $32 \%$ \\
\hline 0,25 & 3,57 & - & \\
\hline$-0,21$ & $-3,55$ & - & \\
\hline 0,22 & 2,88 & - & \\
\hline 0,45 & 6,72 & - & $19 \%$ \\
\hline$-0,25$ & $-3,44$ & - & \\
\hline 0,22 & 3,10 & - & \\
\hline 0,26 & 4,53 & - & $24 \%$ \\
\hline 0,24 & 4,17 & - & \\
\hline 0,16 & 2,37 & 0,02 & $8 \%$ \\
\hline 0,14 & 2,07 & 0,04 & \\
\hline 0,47 & 7,08 & - & $17 \%$ \\
\hline$-0,29$ & $-4,26$ & - & \\
\hline 0,20 & 2,68 & 0,01 & \\
\hline 0,21 & 3,23 & - & $18 \%$ \\
\hline 0,18 & 2,06 & 0,04 & \\
\hline$-0,27$ & $-4,23$ & - & \\
\hline 0,27 & 2,88 & - & \\
\hline 0,27 & 4,28 & - & $19 \%$ \\
\hline 0,19 & 3,03 & - & \\
\hline 0,30 & 5,19 & - & $19 \%$ \\
\hline$-0,24$ & $-3,86$ & - & \\
\hline 0,35 & 4,48 & - & \\
\hline 0,16 & 2,37 & 0,02 & $9 \%$ \\
\hline 0,15 & 2,31 & 0,02 & \\
\hline 0,28 & 4,81 & - & $14 \%$ \\
\hline$-0,20$ & $-3,02$ & - & \\
\hline 0,28 & 3,40 & - & \\
\hline 0,32 & 10,44 & - & $10 \%$ \\
\hline 0,34 & 11,07 & - & $12 \%$ \\
\hline 0,47 & 16,06 & - & $22 \%$ \\
\hline 0,20 & 2,43 & 0,02 & $21 \%$ \\
\hline 0,21 & 2,71 & 0,01 & \\
\hline$-0,32$ & $-4,04$ & - & \\
\hline 0,18 & 2,27 & 0,02 & \\
\hline 0,17 & 2,02 & 0,04 & \\
\hline 0,37 & 5,57 & - & $35 \%$ \\
\hline 0,25 & 3,77 & - & \\
\hline$-0,21$ & $-3,62$ & - & \\
\hline 0,15 & 2,08 & 0,04 & \\
\hline
\end{tabular}

Fonte: Elaboração própria.

Nota: Sinal convencional utilizado:

- Dado numérico igual a zero não resultante de arredondamento.

*Correlação bicaudal significante para $\mathrm{p} \leq 0.05$. 


\section{Discussão}

Os valores de alfa de Cronbach obtidos nas diferentes dimensões do questionário de liderança indicam boa consistência interna da generalidade das dimensões, sendo superiores aos encontrados pelo autor da escala original ${ }^{(21)}$, aos do autor da versão adaptada à area da saúde ${ }^{(17)}$ e aos de outros autores ${ }^{(15,22-23)}$.

A análise da perceção de liderança, com valores médios superiores a 4,5 nas diferentes dimensões, permitiu verificar que os profissionais têm uma imagem moderadamente positiva do exercício da liderança. Não obstante identificarem no seu líder os oito papéis de liderança, os valores médios evidenciam um amplo espaço para o desenvolvimento dos vários papéis de liderança. Os enfermeiros perioperatórios portugueses salientam uma perceção ligeiramente mais elevada nos papéis de Produtor e de Diretor, corroborando resultados de outros estudos ${ }^{(15,17,23)}$. Os líderes, embora desempenhem todos os papéis, têm maior foco na produtividade e no estabelecimento de objetivos. Verifica-se, assim, predominância do modelo dos objetivos racionais, de uma orientação para as relações com o exterior e foco no controlo dos processos. Os enfermeiros perioperatórios percecionam que o líder "Impulsiona a unidade para o cumprimento dos seus objetivos (item 23)", "Verifica se a unidade cumpre os objetivos definidos (item 15)", "Define áreas de responsabilidade para os subordinados (item 7)", bem como "Assegura que todos saibam os objetivos da unidade (item 12)". Esses dados podem refletir o foco dos hospitais de gestão empresarial que são muito vocacionados para os resultados ${ }^{(15)}$. Por outro lado, os enfermeiros perioperatórios demonstram uma perceção menos positiva dos papéis de Inovador e Facilitador, resultados corroborados por outras investigações ${ }^{(15,17)}$. Os participantes percecionam menor capacidade do líder para a gestão da mudança (item 10), integração de inovações (item 25), bem como para ser um pensador criativo (item 1). Competências estas, fundamentais em contextos complexos e em evolução constante, como é o BO. Esses resultados revelam a necessidade de os líderes desenvolverem competências no âmbito do Modelo dos Sistemas Abertos (papel de Inovador), demonstrando maior flexibilidade. Os enfermeiros perioperatórios também observam oportunidades de melhoria na criação de consensos (item 6), na tomada de decisão participativa (item 11), na partilha de ideias de grupo (item 24) e no desenvolvimento do trabalho em equipa (item 31). Tendo em conta que o trabalho no BO desenvolve-se fundamentalmente em equipa e que o trabalho em equipa influencia de forma determinante a SD, é essencial que os líderes também desenvolvam maior foco no Modelo das Relações Humanas, no papel de Facilitador.

Os modelos de regressão dos papéis de liderança com as dimensões do questionário de SDBO permitem salientar que todos os papéis são preditores da SD no BO, com exceção do papel de Broker. Destaca-se o efeito explicativo dos papéis Monitor, Produtor e Facilitador, que entram na maioria dos modelos preditores da SD no BO. Esses resultados enfatizam a importância das competências de liderança para a promoção da SD, nomeadamente no que se refere à capacidade dos líderes em assegurar que as pessoas cumprem as normas e estão a atingir os objetivos, à capacidade de motivar os liderados a aceitar responsabilidades e a manter a produtividade elevada, bem como desenvolver o trabalho colaborativo. O que vai ao encontro das políticas de saúde nacionais, nomeadamente da Estratégia Nacional para a Qualidade em Saúde ${ }^{(24)}$, que define como objetivo estratégico o Reforço da SD, mediante o cumprimento dos objetivos do Plano Nacional de Segurança dos Doentes ${ }^{(2)}$.

Contrariamente, o papel de Mentor tem uma relação inversa com as dimensões da SD, o que nos parece contraditório, tendo em conta que este papel de liderança assenta no desenvolvimento dos liderados, valorizando a escuta ativa e a relação empática. Por outro lado, pode indiciar que a implementação das políticas de segurança ainda está predominantemente ligada ao cumprimento de normas e não em um compromisso coletivo com a segurança. O facto do papel de Broker não ser preditor pode ser explicado por 
representar um papel de um líder mais focado no exercício da influência com o exterior e menos nos processos internos.

O efeito preditor do papel Facilitador, aliado ao facto de ser um dos papéis de liderança percecionado menos positivamente pelos liderados, alerta para a necessidade imperativa dos enfermeiros gestores desenvolverem competências para a promoção do trabalho em equipa e da mediação de conflitos em prol do incremento da SD. Os enfermeiros gestores perioperatórios devem, desse modo, procurar ter maior foco no desenvolvimento de pessoas que permita capacitar os outros profissionais, dando-lhes autonomia e oportunidade de participarem nos processos de decisão, para alcançar consensos e produzir mudanças sustentáveis ${ }^{(25)}$.

A limitação deste estudo deu-se pelo facto de ter sido avaliado apenas o que é característico no líder e não ter sido avaliado o que deveria ser característico na perspectiva dos liderados. Optou-se por desenvolver um estudo de caráter dominantemente quantitativo, mas não se pode deixar de mencionar que esta opção limita a compreensão dos fenómenos.

Os resultados obtidos nesta investigação permitem maior compreensão do exercício da liderança de enfermagem no $\mathrm{BO}$, evidenciando a sua influência na SD, nomeadamente no que se refere aos papéis de liderança mais relevantes nesse contexto. O diagnóstico efetuado ao salientar a perceção dos liderados sobre o exercício da liderança e os papéis preditores da SD, alerta para as áreas prioritárias no desenvolvimento de competências dos líderes.

\section{Conclusão}

Os enfermeiros perioperatórios têm uma perceção moderadamente positiva do exercício da liderança, indicando a necessidade de se promover o desenvolvimento dos diferentes papéis de liderança exercidos pelos enfermeiros gestores perioperatórios portugueses. Não obstante os líderes de enfermagem desempenharem todos os papéis de liderança, identifica-se predomínio dos papéis focados na produtividade, controlo e com orientação para os resultados. Todavia, os resultados evidenciam que, na perspetiva dos enfermeiros perioperatórios, os enfermeiros gestores portugueses devem procurar desenvolver as suas competências na facilitação dos processos de adaptação e mudança, na geração do compromisso e de esforço coletivo dos liderados. Os modelos de regressão revelaram a influência positiva da globalidade dos papéis de liderança na SD no $\mathrm{BO}$, com destaque para os papéis focados nos objetivos, na produtividade e no desenvolvimento do trabalho em equipa.

Considera-se que em estudos futuros seria importante avaliar o gap entre o que é característico da liderança e o que é expectável na perspetiva dos liderados. O desenvolvimento de estudos qualitativos permitiria maior compreensão do processo de liderança e da sua influência na SD.

\section{Colaborações:}

1 - concepção, projeto, análise e interpretação dos dados: Ana Sofia de Carvalho Mota e Amélia Filomena de Oliveira Mendes Castilho;

2 - redação do artigo e revisão crítica relevante do conteúdo intelectual: Ana Sofia de Carvalho Mota, Amélia Filomena de Oliveira Mendes Castilho e Maria Manuela Ferreira Pereira Martins;

3 - aprovação final da versão a ser publicada: Amélia Filomena de Oliveira Mendes Castilho e Maria Manuela Ferreira Pereira Martins.

\section{Referências}

1. World Health Organization. Global Patient Safety Action Plan 2021-2030: Towards eliminating avoidable harm in health care [Internet]. Geneva (CH); 2018 [cited 2021 Sep 30]. Available from: https://www.iapo.org.uk/news/2021/aug/23/ global-patient-safety-action-plan-2021-2030officially-launched

2. Portugal. Ministério da Saúde. Gabinete do Secretário de Estado Adjunto e da Saúde. Despacho n. $.9390 / 2021$. Aprova o Plano Nacional para a Segurança dos Doentes 2021-2026 (PNSD 2021-2026) [Internet]. Diário da República, 
2a série, n. 187. Lisboa (PT); 2021 [cited 2021 Sep 30]. Available from: https://dre.tretas.org/ pdfs/2021/09/24/dre-4672700.pdf

3. World Health Organization. Patient safety: global action on patient safety: report by the Director-General [Internet]. Geneva (CH); 2019 [cited 2021 Sep 30]. Available from: https://apps. who.int/gb/ebwha/pdf_files/WHA72/A72_26-en.pdf

4. World Health Organization. State of the world's nursing 2020: investing in education, jobs and leadership [Internet]. Geneva (CH); 2018 [cited 2021 Sep 30]. Available from: https://apps.who.int/ iris/handle/10665/331677

5. Fagerström L, Kinnunen M, Saarela J. Nursing workload, patient safety incidents and mortality: an observational study from Finland. BMJ Open. 2018;8(4):e016367. DOI:10.1136/bmjopen2017-016367

6. Labrague LJ, De los Santos JAA, Tsaras K, Galabay JR, Falguera CC, Rosales RA, et al. The association of nurse caring behaviours on missed nursing care, adverse patient events and perceived quality of care: A cross-sectional study. J Nursing Manag. 2020;28(8):2257-65. DOI: https://doi.org/ 10.1111/jonm.12894

7. Ducharme MP, Bernhardt JM, Padula CA, Adams JM. Leader Influence, the Professional Practice Environment, and Nurse Engagement in Essential Nursing Practice. J Nurs Adm. 2017;47(78):367-75. DOI: 10.1097/NNA.0000000000000497

8. Boamah SA, Laschinger HKS, Wong C, Clarke S. Effect of transformational leadership on job satisfaction and patient safety outcomes. Nurs Outlook. 2018;66(2):180-9. DOI: https://doi. org/10.1016/j.outlook.2017.10.004

9. Boamah S. Linking Nurses' Clinical Leadership to Patient Care Quality: the Role of Transformational Leadership and Workplace Empowerment. Can J Nurs Res. 2018;50(1):9-19. DOI $10.1177 / 0844562117732490$

10. Adams JM, Djukic M, Gregas M, Fryer AK. Influence of Nurse Leader Practice Characteristics on Patient Outcomes: Results from a Multi-State Study. Nurs Econ [Internet]. 2018 [cited 2021 Sep 30];36(6):259-67. Available from: https:// www.nursingeconomics.net/necfiles/2018/ND18/ 259.pdf

11. Organização Mundial de Saúde. Orientações da OMS para Cirurgia Segura 2009: Cirurgia Segura Salva Vidas [Internet]. Lisboa (PT); 2009 [cited
2021 Set 30]. Available from: https://www.dgs.pt/ ficheiros-de-upload-2/orientacoes-da-oms-para-acirurgia-segura-2009-pdf.aspx

12. De Vries EN, Ramrattan MA, Smorenburg SM, Gouma DJ, Boermeester MA. The incidence and nature of in-hospital adverse events: a systematic review. Qual Saf Health Care. 2008;17(3):216-23. DOI:10.1136/qshc.2007.023622

13. Halfon P, Staines A, Burnand B. Adverse events related to hospital care: a retrospective medical records review in a Swiss hospital. Int J Qual Health Care. 2017;29(4):527-33. DOI: https://doi. org/10.1093/intqhc/mzx061

14. Quinn RE, Rohrbaugh J. A Spatial Model of Effectiveness Criteria: Towards a Competing Values Approach to Organizational Analysis. Manage Sci [Internet]. 1983 [cited 2021 Sep 30];29(3):363-77. Available from: http://www.jstor. org/stable/2631061

15. Melo RC, Mónico LS, Carvalho C, Parreira PS. A liderança de enfermagem nas organizações de saúde. In: Pereira P, Mónico L, Carvalho C, organizadores. Liderança e Seus Efeitos. Coimbra (PT): Escola Superior de Enfermagem de Coimbra; 2017. p. 7-25.

16. Quinn RE, Thompson MP, Faerman SR, McGrath M. Competências gerenciais: princípios e aplicações. 3a ed. Rio de Janeiro: Elsevier; 2003.

17. Parreira PM, Felício MJ, Lopes A, Nave FJGM, Parreora F. Papéis de liderança: um instrumento avaliativo. RIE [Internet]. 2006 [cited 2021 Sep 30];13:3-14. Available from: https://www. researchgate.net/publication/284818605_Papeis_ de_lideranca_um_instrumento_avaliativo

18. Mota ASC, Castilho AFOM. Construção e validação psicométrica do Questionário de Segurança do Doente no Bloco Operatório. Rev Enf Ref. 2019:21(2):67-78. DOI: https://doi.org/10.12707/ RIV19012

19. Mota ASC, Castilho AFOM, Martins MMFPS. Assessment of patient safety in the operating room: nurses' perceptions. Rev Enf Ref. 2021;5(6):1-10. DOI: https://doi. org/10.12707/RV20134

20. Portugal. Ministério da Saúde. Avaliação da situação nacional dos blocos operatórios: relatório final [Internet]. Lisboa (PT); 2015 [cited 2021 Sep 30]. Available from: http://www.apca.com.pt/ documentos/2015/Avaliacao_situacao_nacional_ blocos_operatorios_Outubro2015.pdf 
12

Papéis de liderança em enfermagem preditores da segurança do doente no bloco operatório

21. Quinn RE. Beyond Rational Management: Mastering the Paradoxes and Competing Demands of High Performance. San Francisco (US): JosseyBass Inc; 1988.

22. Parreira P, Lopes A, Salgueiro F, Carvalho C, Oliveira AS, Castilho A, et al. Papéis de liderança de Quinn: um estudo realizado em Serviços de Saúde Portugueses com recurso à análise fatorial confirmatória. Rev Ibero-Americana de Saúde e Envelhecimento. 2015;1(2):192-217. DOI:10.24902/r.riase.2015.1(2).192

23. Castilho AF. Eventos Adversos nos Cuidados de Enfermagem ao Doente Internado: contributos para a Política de Segurança [tese]. Porto: Instituto de Ciências Biomédicas Abel Salazar da Universidade do Porto; 2014
24. Portugal. Ministério da Saúde. Despacho no 5613/2015, de 27 de maio de 2015. Aprova a Estratégia Nacional para a Qualidade na Saúde 2015-2020 [Internet]. Lisboa (PT); 2015 [cited 2021 Sep 30]. Available from: https://dre.pt/web/guest/ pesquisa/-/search/67324029/details/normal?1=1

25. Durmuş SÇ, Kırca K. Leadership Styles in Nursing. In: Durmuş SÇ, editor. Nursing-New Perspectives. London: IntechOpen. DOI: 10.5772/ intechopen.89679 2019

Recebido: 30 de setembro de 2021

Aprovado: 3 de novembro de 2021

Publicado: 22 de novembro de 2021

A Revista Baiana de Enfermagem utiliza a Licença Creative Commons - Atribuição-NãoComercial 4.0 Internacional.

https://creativecommons.org/licenses/by-nc/4.0/

Este artigo é de acesso aberto distribuído sob os termos da Licença Creative Commons (CC BY-NC).

Esta licença permite que outros remixem, adaptem e criem a partir do seu trabalho para fins não comerciais.

Embora os novos trabalhos tenham de lhe atribuir o devido crédito e não possam ser usados para fins comerciais, os usuários não têm de licenciar esses trabalhos derivados sob os mesmos termos. 